\title{
Mimics: \\ A symbolic conflict/cooperation simulation program, with embedded protocol recording and automatic psychometric assessment
}

\author{
EUGENE V. AIDMAN \\ University of Ballarat, Mt. Helen, Victoria, Australia \\ and \\ ALEXANDER G. SHMELYOV \\ Moscow State University, Moscow, Russia
}

\begin{abstract}
This paper describes an interactive software environment designed as a social interaction simulator with embedded comprehensive recording and flexible assessment facilities. Using schematized visual sketches similar to cross-cultural facial universals (Ekman, 1999), Mimics (Shmelyov \& Aidman, 1997) employs a computer-game-like scenario that requires the subject to identify with an avatar and navigate it through a playing field inhabited by hosts who display a range of facial expressions. From these expressions (which are highly consequential), the player has to anticipate the hosts' reactions to the avatar (which may vary from friendly to obstructing or aggressive) and choose between negotiating with a host (by altering the avatar's facial expression), attacking it, or searching for an escape route. Comprehensive recording of player moves and interactions has enabled computation of several finegrained indices of interactive behavior, such as aggressive response styles, efficiency, and motivation in conflict/cooperation contexts. Initial validation data and potential applications of the method in the assessment of personality and social behavior are discussed.
\end{abstract}

Computer game technologyoffers an appealing paradigm for the development of models for behavioral research and assessment (Case, 1995; Porter, 1995). Both existing commercial games (e.g., Donchin, 1995; Gonzalez \& Cathcart, 1995) and purpose-developed game-like software (e.g., Allan, 1995; Ryan, 1994; Washburn \& Gulledge, 1995) have been utilized in several attempts to examine cognitive functions such as memory (Ryan, 1994; Washburn \& Gulledge, 1995), skill acquisition (see Donchin, 1995), and strategy development (Gonzalez \& Cathcart, 1995).

The Mimics software is an attempt to apply the principles of computer-game-embedded measurement to the assessment of personality and social behavior in contexts of potential interpersonal conflict. We were particularly motivated by the prospect of minimizing self-presentation ef-

The development of this software was supported by Human Technologies (Moscow) and InterMind Consulting (Melbourne). The authors thank D. Bondarenko and A. Frolov for their $\mathrm{C}++$ programming contribution. We are also grateful to Marijke Heywood for computer graphics advice, to Gary Schober for assistance with data collection, and to Michael Lake for his comments on the earlier versions of the paper. The current version of Mimics, as described in this article, has not been commercialized. The authors may, however, have a financial interest in its future development. Correspondence concerning this article should be directed to E. V. Aidman, Senior Research Scientist, LOD, Defence Sciences and Technology Organisation, P. O. Box 1500, Edinburgh, SA 5111, Australia (e-mail: eugene.aidman@dsto.defence.govau). fects in the assessment of individual choice in such contexts. Game-like simulations, such as Prisoner Dilemma games (Luce \& Raiffa, 1957; Pizzorno, 1991), have been common in the experimental investigation of conflict/ cooperation choice in social interaction. The ecological validity of the Prisoner Dilemma paradigm, however, is limited by the fact that the player is forced to choose from an artificially constrained range of behavioral strategies. Computer and video games may offer more complex and realistic scenarios where multiple strategies of conflict/ cooperation choice can be studied (see Allan, 1995; Case, 1995; Donchin, 1995). The value of such an approach is reaffirmed by the prospects of psychologicaltesting in virtual reality, where interpersonal interaction is cited among the most immediate and attractive assessment targets (FernandezBallesteros, 1994).

The idea of measuring complex cognitive functioning through synthetic environments and simulations is not new (Chiles, 1982; Fischetti \& Truxal, 1985). The promise of the early systems - such as the Strategic and Tactical Assessment Record (STAR; Graham, Cook, Cohen, Phelps, \& Gerkovich, 1985)—was that combining simulated environments with embedded performance assessment techniques would provide unobtrusive behavioral measures of integrated task performance, with a minimum of performance interference attributable to the measurement itself. Indeed, STAR produced an impressive array of opera- 
tionally defined perceptual and information-processing parameters, including risk-taking behavior, comparable with common laboratory measurements. One of its key advantages, according to Graham et al., was that "all measurement procedures were embedded within the operations required to play a computer game" (p. 643), which made them both unobtrusive and highly motivating. However, subsequentimplementations of this approach have been relatively narrow (see Siem, 1992) and have generated substantial problems with meaningful operationalization of measurement constructs (Porter, 1995) and inherent conflict between the "open-endedness" and multidimensionality (Donchin, 1995) intrinsic in the typical computer game, as well as with the task of establishing the controlled conditions that are required for measurement (Case, 1995).

The purpose of the present report is to describe a software environment that implements the principles of computer-gameembedded measurement in the context of the quantification of interactional styles and to discuss its potential applications in the assessment of personality and social behavior.

\section{DESCRIPTION OF MIMICS}

Mimics is an interactive software environment designed as a social interaction simulator with embedded comprehensive protocol recording facilities. Visually, Mimics is based on schematized cross-cultural facial universals (Ekman, 1992,1999), covering a range of simple and readily recognizable expressions. Operationally, its objective is to elicit and assess strategic choice and tactical responses in a conflict/cooperation choice paradigm (Aidman, 1999). The central component of the Mimics software system is the game, Mimix (Shmelyov \& Aidman, 1997), which provides a computer-game-like scenario in which the subject (the player) is required to control schematic facial expressions of the avatar (the brighter face on Figure 1) and navigate a playing field containing cells filled with hosts (the darker faces on Figure 1), who display expressions from the same range as the avatar's. Figure 1 shows what the visible part of the playing field looks like on screen; its actual size is $9 \times 9$ cells per level of game, with a $3 \times 3$ segment visible at any one time.

The hosts' reactions to the avatar depend on both their and the avatar's expressions and range from friendly and supportive to obstructing or even expressly aggressive. Host responses are accompanied with relevant visual and sound effects, thus supporting more accurate and realistic perception of those responses by the player. The changeable features of the avatar's face are the mouth, the eyes, and the eyebrows. Each can be made smiling, neutral, or frowningindependently of the status of the other two features-by a single keypress, following an intuitive combination of keys on the keyboard (see Figure 2). The hosts' facial expressions follow the same $3 \times 3 \times 3$ combinatorics, thus providing 27 possible different expressions, which is minimally sufficient to discourage players from using shortterm memory and, instead, encourage them to approach
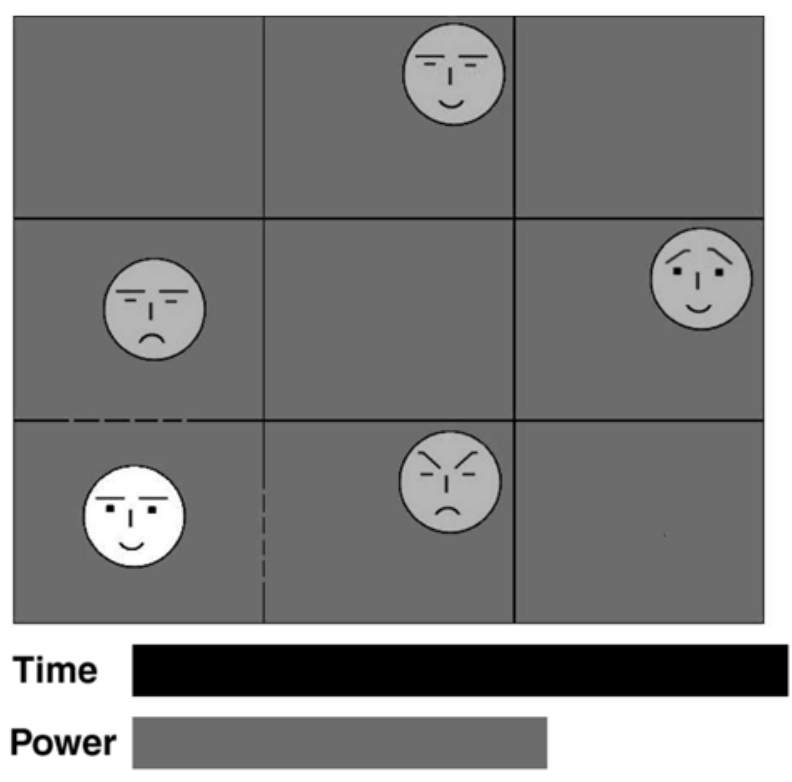

Figure 1. A sketch of a typical Mimics screen. The brighter face in the bottom left corner is the avatar; the darker faces are hosts.

individual interactions as problem-solving rather than memory tasks.

The player is given limited time and power resources to move the avatar through the hosts' cells and reach the desired destination (the top of the playing field) to collect the reward (a symbolic apple, plus additional power and entry to the next level of the game). Both resource indicatorstime and power-are prominently displayed at the bottom of the screen (see Figure 1). As a motivating factor in the game, scoring points are given for each individual hostavatar interaction, depending on how well the player anticipates the host's response and adjusts the avatar's expression before engaging with the host. The top 10 scorers are presented in a Table of Records, thus encouraging the competitiveness of the game. However, when the game is used for assessment purposes, the Table of Records is cleared of all records other than those of the current player.

Below is a brief summary of the game scenario offered to the Mimix player (adapted from Aidman, 1999; see Figure 2 for more detail):

Imagine yourself in charge of an avatar so that you can put on a smile or a frown on its "virtual face," as well as several other expressions, as you choose. You will be treading your way through a maze of virtual rooms inhabited by hosts who may help, block, or even attack you-depending on how they like your avatar's facial expression. You will try to predict the hosts' responses from their expressions and change your own expression to make them let you through. If unsuccessful, go one step back and try another expression or try another route. You can even punch the host if nothing else works. But a happy host may give extra power for your journey. 


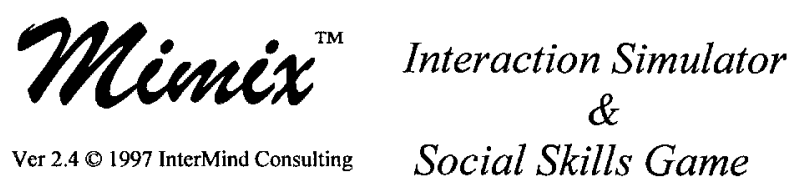

Player Instructions

Use arrow keys to guide your Avatar (a bright face) to the topright corner of the screen. Collect a reward apple at top of each level, which is a 9x9 board scrolling on screen so you can see a portion $-3 \times 3$ cells.

Each cell has a Host who may help, block or even attack you, depending on how they like you. Try to predict the Host's response from their expression and adjust your own expression to make them let you through.

If unsuccessful - go one step back and try another expression or try another route. If nothing works - you may punch the host by holding down SPACE BAR on entrance to their cell (it will cost you power, though). But if you please them - you may get extra power.

Remember, your expression can only change outside host cells. Use the following keys:

\begin{tabular}{|c|c|c|c|}
\hline & Frowning & Neutral & Smiling \\
\hline Brows & $\mathbf{Q}$ & $\mathbf{W}$ & $\mathbf{E}$ \\
\hline Eyes & $\mathbf{A}$ & $\mathbf{S}$ & $\mathbf{D}$ \\
\hline Mouth & $\mathbf{Z}$ & $\mathbf{X}$ & $\mathbf{C}$ \\
\hline
\end{tabular}

The game gets tougher as you progress and ends only when you run out of time or power.

\section{see how far you can go!}

Figure 2. Mimics player instructions. From Mimics: Administration and Interpretation Manual (p. 11), by E. V. Aidman, 1999, Melbourne: InterMind. Copyright 1999 by InterMind Consulting. Adapted with permission.

The objective of the game is, therefore, twofold: to reach the desired destination (top of the level) and to score maximum possible points along the way. The objective can be achieved through any combination of the following three tactics: (1) searching for effective expressions, (2) searching for efficient routes, and (3) attacking the hosts.

The player is free to choose between these three main tactics in any individual interaction. Mimics can thus be seen as a form of projective game (Heiss, 1964): There is a number of scoring options, no prompting at any stage, and the standard player instructions emphasize that players are left to themselves in selecting their preferred scoring tactics. To ensure that this choice is fully informed, how- ever, the embedded scoring engine utilizes an algorithm that automatically monitors player tactics during practice trials. The algorithm triggers the psychometric scoring as soon as the player has used each of the available tactics at least once. The critical move the algorithm is tuned for is attacking the host (by holding the space bar down immediately upon entering the interaction). Hence, the critical stage for the player is moving past the "fatally aggressive" hosts who would always attack the avatar, no matter what its expression. There is no other way of getting the first reward at the end of the first level, other than through attacking these "aggressors." Getting the first reward token (an apple) usually means that the player has "sampled" all 
the tactics available in the game, and from this point on, his/her choice of moves is assumed to be fully informed. The behavior sampling for the subsequent psychometric scoring begins exactly at this point and requires the player to finish the current game and then play three more games, in order to complete the minimum data set sufficient for scoring purposes.

\section{PROGRAM OPERATION}

Mimics software is written for a DOS environment with minimal graphic requirements: Mimics can even be used on an old 386 PC with a CGA graphic adaptor, and the whole package takes less than 100 kilobytes of disk space. The software contains two main components: (1) the game module MIMIX.EXE (written in $\mathrm{C}++$ for DOS) and (2) the scoring and interpretation module MIMICINT.EXE (written in Pascal).

The current version of Mimics was not intended as an integrated system: The MIMIX game is a stand-alone module that can be used without pursuing any assessment purposes (there are consistent indications that many users find it beneficial "as is"- - a useful simulator and an enjoyable game at the same time). In addition, however, for each player MIMIX automatically generates a full record of playing actions in a protocol readable by the scoring module MIMICINT. The records of all subsequent games for the same player are appended to the player's data file, no matter when these are played, unless the player changes his or her code name (a standard DOS file name up to eight characters long), which has to be entered before each game.

A full description of each move in the game is recorded in hexadecimal format and stored in a binary file, assigned the player's code name, for future analysis by the scoring module MIMICINT. The description includes the codes for both the avatar's and the host's expressions, as well as a code for the resulting interaction. Such detailed and comprehensive recording of the player's moves and interactions has provided for computation of a number of finegrained behavioral indices, and it allows for their further refinement, as well as for the development of new indices.

\section{MEASUREMENT OUTPUT}

Currently, the scoring module computes 26 indices, including measures of performance efficiency, interactional styles, and motivation (see Table 1 for a selection of indices and their operational definitions). In particular, the measures of interactional style include a distinct group of indices that reflect players' propensity to hostile/aggressive responses, such as the unprovoked attacks (aggression as an intrinsic choice), retaliatory attacks (aggression mirroring), and frustration-driven attacks (aggressive overreaction to blockings), as well as tendencies to intrapunitive and avoidant responding to aggression - the choice of a frowning expression (threatening) or the search for new routes (evasion) after suffering an attack. More general indices are also computed, such as spontaneousactivity, expression/ route diversity (the number of different expressions tried and number of different hosts visited), and determination (sustaining the game after a critical loss of power).

In the study that follows, we investigated the validity of these new indices for the measurement of individual differences in propensity toward aggression. We examined, first, the associations between the choice of various attacking actions while playing Mimics and self-reported aggressiveness and, second, whether attacking, intropunitive, and avoidant response patterns in Mimics could be predictably influenced by an aggression-inducing instruction.

\section{AGGRESSIVE RESPONSE IN MIMICS AND SELF-REPORTED AGGRESSION}

\section{Method}

The empirical study employed two cohorts of psychology undergraduates (modal age, 19 years) enrolled at a regional Australian university, who participated for optional course credit. In the first cohort, a group of 45 undergraduates played Mimics by following a standard neutral instruction that neither concealed nor emphasized the availability of the attacking option to the player. Upon completion of the game, the subjects responded to Buss and Perry's (1992) Aggression Questionnaire and to a self-report version of Björkqvist, Lagerspetz, and Österman's (1992) Survey of Indirect Aggression. Cronbach's alphas ranged between .8 and .92 for the Buss-Perry subscales (Buss \& Perry, 1992) and .74-.89 for the Bjorkvist et al. instrument, indicating good internal consistency of the instruments' subscales.

The second group of subjects $(N=39)$ played Mimics with an "open" instruction, which emphasized the availability of the attacking option and indicated that it could be used as much as they liked. This "open" instruction effectively created a condition of play in which aggressive responses were encouraged; it will be referred to as the aggressive condition.

\section{Results}

Self-reported aggression and attacking behavior in Mimics. The pattern of correlations, presented in Table 2, is generally supportive of the initial validity of Mimics' measures. Despite the absence of significant correlations between self-reported aggression measures and the indices of unprovoked and retaliatory attacks, predictable and consistent correlations with self-reported aggression were produced by the measure of frustration-driven attacks, aggressive overreaction to blockings: It correlated at .35 ( $p<.01)$ with Björkqvist et al.'s (1992) physical aggression score and at .26 and .24 with Buss and Perry's (1992) anger and physical aggression scores, respectively $(p<.05$, in both cases). Most interesting, a relatively passive form of aggressive response in Mimics - threateningcorrelated with Buss and Perry's verbal aggression score $(r=.31, p<.025)$ and showed a near-significant tendency to correlate with Buss and Perry's anger and hostility scores $(r=.18, p=.08$, in both cases).

Player response under neutral and aggressive conditions. As can be seen from Table 3, the two instructioninduced conditions produced significant group differences in player response measures: Multivariate differ- 
Table 1

Selected Player Strategy Parameters Measured From the Mimics Protocol

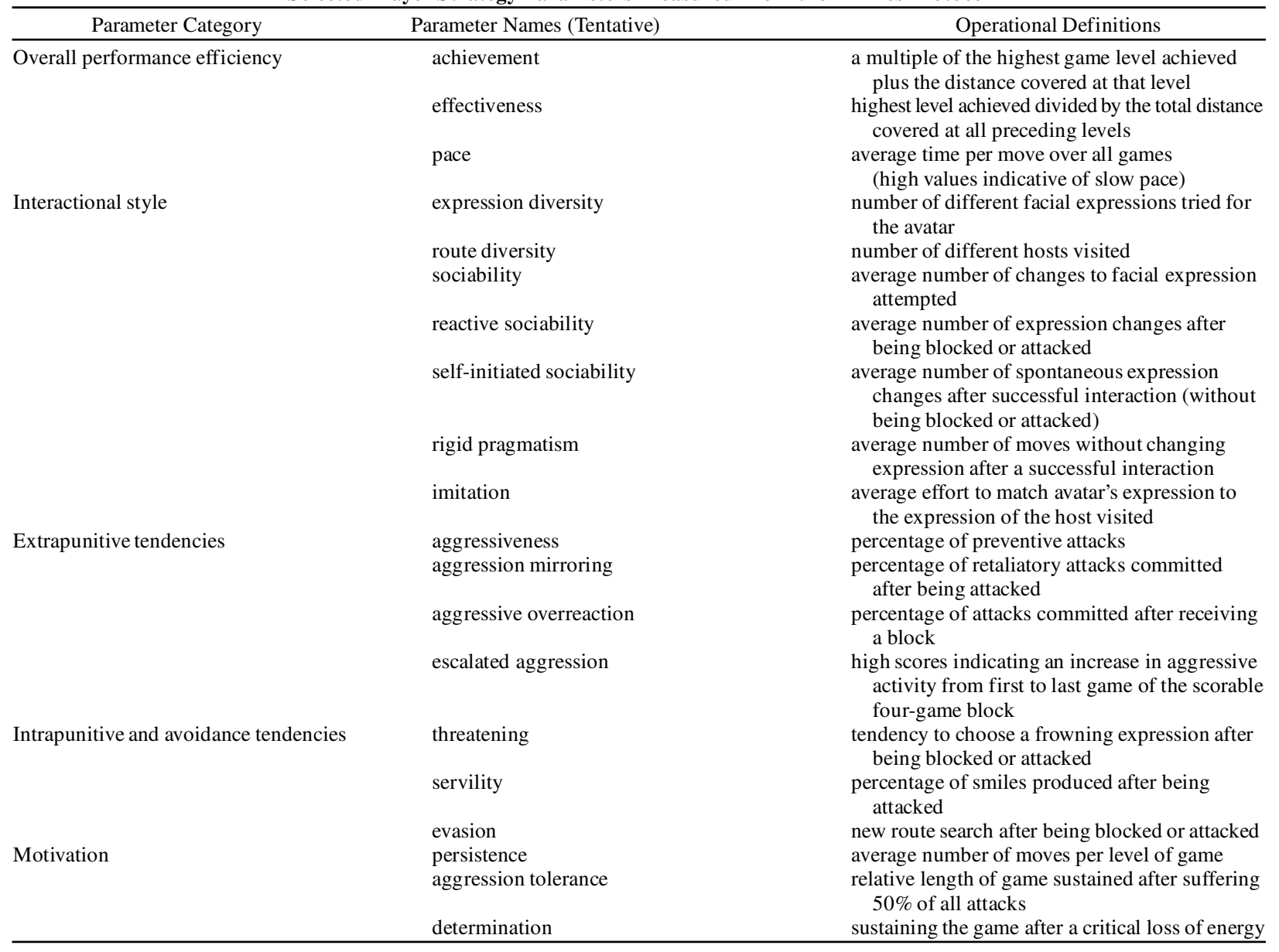

ences were highly significant $[F(5,78)=3.74, p<.005]$. Subsequent univariate analyses showed that, as compared with the group who played in the neutral condition, the aggressive condition group showed significantly higher rates of unprovoked attacks $(t=5.80, p<.001)$ and frustration-driven attacks $(t=3.48, p=.001)$, whereas their rates of evasion and threatening responses were significantly lower $(t=-4.90, p<.001$, and $t=-1.99, p=.05$, respectively). Interestingly, the groups did not differ in the rate of retaliatory attacks $(t=0.16, p=.87)$, indicating that "defensive" attacking — that is, in response to being attacked - may be resistant to aggression-inducing instructions.
Overall, these results support the initial validity for the indices of attacking, threatening, and evasion moves, computed in Mimics, as measures of extrapunitive and avoidant response patterns. Further validation and reliability analysis is currently underway (see Aidman, 2000).

\section{APPLICATIONS}

Mimics appears to be a promising tool, with a number of potential applications in two different forms. First, the automated behavior observation and assessment module, embedded in Mimics, produces a number of useful behav-

Table 2

Correlations Between Mimics' Measures and Self-Reported Aggression $(N=45)$

\begin{tabular}{|c|c|c|c|c|c|c|c|c|}
\hline \multirow[b]{3}{*}{ Mimics' Measures } & \multicolumn{4}{|c|}{ Björkqvist, Lagerspetz, andÖsterman (1992) } & \multirow{2}{*}{\multicolumn{4}{|c|}{ Buss and Perry (1992) }} \\
\hline & & & Indirect & Indirect & & & & \\
\hline & Physical & Verbal & Social & Rational & Physical & Verbal & Anger & Hostility \\
\hline Unprovoked attacks & .15 & .04 & -.09 & -.17 & -.1 & -.17 & -.03 & -.18 \\
\hline Retaliatory attacks & -.14 & -.07 & .15 & -.09 & -.20 & -.18 & -.8 & -.01 \\
\hline Aggressive overreaction & $.35^{\dagger}$ & .05 & -.22 & -.19 & $.24 *$ & -.04 & $.26 *$ & .02 \\
\hline Threatening & .10 & .13 & .04 & .15 & .17 & $.31 *$ & .18 & .18 \\
\hline
\end{tabular}

\footnotetext{
${ }^{*} p<.05 . \quad{ }^{\dagger} p<.01$.
} 
Table 3

Player Response Measures: Means and Standard Deviations for the Groups in the Neutral $(N=45)$ and Aggressive $(N=39)$ Instruction Conditions

\begin{tabular}{|c|c|c|c|c|c|c|}
\hline \multirow[b]{2}{*}{ Mimics' Measures } & \multicolumn{2}{|c|}{ Neutral Instruction } & \multicolumn{2}{|c|}{ Aggressive Instruction } & \multicolumn{2}{|c|}{ Comparison } \\
\hline & $M$ & $S D$ & $M$ & $S D$ & $t$ & $p$ \\
\hline Unprovoked attacks & 15.7 & 8.8 & 26.8 & 9.4 & 5.80 & $<.001$ \\
\hline Retaliatory attacks & 47.8 & 28.1 & 48.9 & 42.8 & 0.16 & .871 \\
\hline Aggressive overreaction & 19.6 & 32.8 & 45.1 & 39.4 & 3.48 & .001 \\
\hline Threatening & 6.7 & 7.6 & 3.7 & 6.9 & -1.99 & .05 \\
\hline Evasion & 12.0 & 8.8 & 3.8 & 6.5 & -4.90 & $<.001$ \\
\hline
\end{tabular}

ioral indices that not only can be used as transparent experimental measures of interactive responses, but as the data reported above indicate, may form the basis for standardized psychometric assessment capable of providing the player with feedback on their social interaction performance and associated personality traits.

Second, as a stand-alone game, it can be used as a social interaction simulator that enables the user to combine the development of social skills with an enjoyable process of playing in a computer-game-like environment. An important feature of Mimics as a social interaction simulator is that it introduces concepts of resource allocation and management in social interaction through a process that could be classified as implicit learning (Kihlstrom, 1994; Stadler \& Frensch, 1998), thus developing some important new tacit knowledge (Reber, 1989; Sternberg, 1997): Without any explicit instruction, the player is effectively encouraged to account for his or her time and power resources in negotiating the avatar's interactions with the hosts. More than half of the 84 subjects $(55.9 \%)$ in the study reported here admitted that they had never considered the role of resource economy in their past negotiations. Another pilot study showed that subjects consistently reported their playing actions to have been strongly influenced by the amount of time and power they had remaining at the time (Aidman, 2000). A preliminary conclusion is, therefore, that Mimics may encourage reflection and metacognitive strategies related to social interaction.

\section{PROGRAM AVAILABILITY AND EQUIPMENT REQUIREMENTS}

The current version of the game module Mimix can be downloaded at www.ballarat.edu.au/intermind at no cost. The psychometric assessment module Mimix-Pro is available to qualified users for research, evaluation, and other noncommercial uses; requests should be e-mailed to the first author. Mimics will work on any PC driven by DOS 5.01 or higher. It has been tested under Windows 3.x, 95 , and 98; however, DOS emulation mode is recommended for Mimix: The game module was designed to work in full screen only, and as such, it will disable (or conflict with) most window-resizing functions. A fully integrated, Windows version of the program is currently under construction. When ready, it will enable flexible, on-line administration and data collection, with data formats readable in most spreadsheet and data analysis packages.

\section{REFERENCES}

Aidman, E. V. (1999). Mimics: Administration and interpretation manual. Melbourne: InterMind.

AIDMAN, E. V. (2000, July). Computerised interaction simulation in the assessment of aggression-related response style and dispositions. Paper presented at the XIV World Meeting of the International Society for Research on Aggression, "Prevention and Control of Aggression and the Impact on its Victims," Valencia, Spain.

Allan, R. W. (1995). The matching game: Testing for the generality of the matching law. Behavior Research Methods, Instruments, \& Computers, 27, 206-210.

Bü̈rkqVist, K., Lagerspetz, K. M. J., \& Österman, K. (1992). The direct and indirect aggression scales. Åbo, Finland: Åbo Akademi University, Department of Social Sciences.

Buss, A. H., \& Perry, M. (1992). The aggression questionnaire. Journal of Personality and Social Psychology, 63, 452-459.

CASE, D. A. (1995). On trekking to operant frontiers. Behavior Research Methods, Instruments, \& Computers, 27, 211-216.

Chiles, W. D. (1982). Workload, task and situational factors as modifiers of complex human behavior. In E. A. Alluisi \& E. A. Fleishman (Eds.), Human performance and productivity: Vol. 3. Stress and performance effectiveness (pp. 11-56). Hillsdale, NJ: Erlbaum.

Donchin, E. (1995). Video games as research tools: The Space Fortress game. Behavior Research Methods, Instruments, \& Computers, 27, 217-223.

EKMAN, P. (1992). Facial expressions of emotion: An old controversy and new findings. In V. Bruce \& A. Cowey (Eds.), Processing the facial image (pp. 63-69). Oxford: Oxford University Press, Clarendon Press.

EkMAN, P. (1999). Facial expressions. In T. Dalgleish \& M. J. Power (Eds.), Handbook of cognition and emotion (pp. 301-320). Chichester, U.K.: Wiley.

Fernandez-Ballesteros, R. (1994). Psychological assessment. Applied Psychology: An International Review, 43, 157-175.

Fischetti, M. A., \& Truxal, C. (1985). Computers simulating "the right stuff.' IEEE Spectrum, 22, 38-47.

Gonzalez, F. A., \& CAThcart, M. (1995). A procedure for studying strategy development in humans. Behavior Research Methods, Instruments, \& Computers, 27, 224-228.

Graham, G., Cook, M., Cohen, M., Phelps, M., \& Gerkovich, M. (1985). STAR: A unique embedded performance assessment technique. Behavior Research Methods, Instruments, \& Computers, 17, 642-651.

HeIss, R. (Ed.) (1964). Handbuch der Psychologie: Psychologische Diagnostik. Göttingen: Verlag für Psychologie.

Kinlstrom, J. F. (1994). Cognition unawares. Science, 264, 1013-1016.

Luce, R. D., \& RAIfFA, H. (1957). Games and decisions. New York: Wiley.

Pizzorno, A. (1991). On the individualistic theory of social order. In P. Bourdieu \& J. S. Coleman (Eds.), Social theory for a changing society (pp. 209-244). New York: Russell Sage Foundation.

Porter, D. B. (1995). Computer games: Paradigms of opportunity. Behavior Research Methods, Instruments, \& Computers, 27, 229-234.

REBER, A. S. (1989). Implicit learning and tacit knowledge. Journal of Experimental Psychology: General, 118, 219-235.

Ryan, E. B. (1994). Memory for Goblins: A computer game for assessing and training working memory skill. Computers in Human Services, 11, 213-217. 
Shmelyov, A., \& Aidman, E. (1997). MIMICS: Interaction simulator and social skills game [Computer software]. Melbourne: InterMind. Siem, F. M. (1992). Predictive validity of an automated personality inventory for Air Force pilot selection. International Journal of Aviation Psychology, 2, 261-270.

Stadler, M. A., \& Frensch, P. A. (Eds.) (1998). Handbook of implicit learning. Thousand Oaks, CA: Sage.
Sternberg, R. J. (1997). Successful intelligence. New York: Plume. WAshburn, D. A., \& GULledGe, J. P. (1995). Game-like tasks for comparative research: Leveling the playing field. Behavior Research Methods, Instruments, \& Computers, 27, 235-238.

(Manuscript received April 28, 2000; revision accepted for publication August 5, 2001.) 